\title{
De la genèse du nouveau Conseil National de la Comptabilité (2007) : un cas d'isomorphisme institutionnel ?
}

\author{
Bernard Colasse et Christine Pochet
}

\section{Résumé}

Cet article propose une interprétation de la réforme du dispositif français de normalisation initiée par le décret n 2007-629 du 27 avril 2007 relatif au Conseil National de la Comptabilité (CNC). Cette réforme, si elle menée à son terme, doit déboucher sur une Autorité (française) des Normes Comptables (ANC).

Cette interprétation relève de l'analyse néo-institutionnelle. Elle mobilise en particulier les notions de «dépendance de sentier » et de "mimétisme » institutionnel. Le nouveau CNC est d'abord situé en tant qu'institution par rapport à ses prédécesseurs. Il est ensuite comparé à deux institutions dont l'architecture a pu inspirer ses concepteurs, le Financial Accounting Standards Board (FASB) et l'Autorité des Marchés Financiers (AMF).

Il apparaît que le nouveau CNC s'écarte fortement du sentier de la normalisation française et se rapproche des autorités administratives indépendantes du type AMF. Or, ces autorités sont fortement inspirées de la Securities and Exchange Commission (SEC) américaine. Le modèle de la nouvelle ANC serait donc davantage la SEC que le FASB.

Mots-clés : Institutions de normalisation - Normalisation comptable française Régulation comptable - Conseil National de la Comptabilité ;

\begin{abstract}
This article proposes an interpretation of the reform of the French accounting standards setter initiated by the decrete $n^{\circ} 20076629$ of the $27^{\text {th }}$ of april 2007 related to the national standards board, the Conseil National de la Comptabilité (CNC). This reform, if it goes to its term, will give birth to a French Autorité des Normes Comptables (ANC).

The proposed interpretation derives from a neo-institutionalist framework. It uses in particularly the notions of path dependency and of institutional mimetism. First, the new CNC is situated as an institution in relation with its predecessors. Then, It is compared with two institutions the architecture of which could have inspired its conceptors, the Financial Accounting Standards Board (FASB) and the French Autorité des Marchés Financiers (AMF).

It appears that the new CNC clearly steps out of the the French accounting regulation's historical path and tends to mime French "autorités administratives indépendantes" of the AMF type. Now, such an "autorite" is highly inspired from the SEC. So, paradoxically, the model of the new French Autorité des Normes Comptables would be the SEC rather than the FASB.
\end{abstract}

Keywords: Accounting standards setting institutions - French accounting standardization - Accounting regulation - French Accounting Board. 
Remerciements : Les auteurs remercient chaleureusement M. Jean-François Lepetit pour le long entretien qu'il a bien voulu leur accorder, M. Jean-Paul Milot, ancien secrétaire général du CNC, pour ses commentaires avisés d'une première version de cet article et, enfin, les deux rapporteurs anonymes pour leurs suggestions très constructives d'amélioration.

\section{Correspondance :}

Bernard Colasse

DRM-CREFIGE

Université Paris-Dauphine

colasse@crefige.dauphine.fr;
Christine Pochet

GREGOR

IAE de PARIS

christine.pochet@univ-paris1.fr

\section{Introduction}

Sans doute parce que l'information comptable participe très directement au fonctionnement des marchés financiers et du système capitaliste, sa normalisation est devenue un enjeu de première importance pour les pouvoirs publics. Tant qu'elle est restée circonscrite à l'échelle nationale, la normalisation comptable pouvait être assez facilement contrôlée par l'autorité publique. Il en va tout autrement depuis l'apparition d'une gouvernance comptable à plusieurs niveaux (multi-level governance). Les efforts d'harmonisation internationale ont en effet débouché sur la création d'un organisme de droit privé, l’IASB (International Accounting Standards Board) dont les normes s'imposent depuis le $1^{\mathrm{er}}$ janvier 2005 aux Etats-membres de l’Union Européenne. Dans ce contexte, le rôle des normalisateurs nationaux a évolué, particulièrement au sein de l'UE où ces derniers sont le plus souvent impliqués dans le processus d'incorporation des normes internationales dans le droit communautaire via la procédure de comitologie.

Tous les grands pays s'efforcent donc de participer à cette normalisation et de se doter d'institutions aussi « performantes » que possible en la matière. C'est le cas de la France qui, moins d'une dizaine d'années après la précédente, a lancé une nouvelle réforme de son dispositif de normalisation.

En effet, en pleine campagne présidentielle française, le 27 avril 2007, a été publié un décret qui modifie le Conseil National de la Comptabilité (CNC).

Selon son principal promoteur, Monsieur Jean-François Lepetit ${ }^{1}$, cette réforme devrait déboucher à plus ou moins brève échéance sur la création d'une Autorité des Normes Comptables (ANC) française qui exercerait les missions exercées actuellement 
respectivement par l'actuel CNC, né d’un décret de 1996, et le Comité de la Réglementation Comptable, créé par une loi de $1998^{2}$.

Dans cet article, nous tenterons de contribuer à l'intelligibilité de ce nouveau CNC et de la future ANC et, plus généralement, à celle des systèmes de normalisation. Plusieurs travaux ont été consacrés récemment à l'analyse du processus de normalisation comptable dans sa dimension internationale (Botzem et Quack, 2006 ; Chiapello et Medjad, 2007 ; Djelic et Quack, 2007). Nous nous situons pour notre part à un niveau d'analyse différent, celui du normalisateur national, dans l'esprit des travaux de Colasse et Standish (1998), ChantiriChaudemanche (2004) pour la France et de Young (1994) pour le cas des Etats-Unis.

Dans une première section, nous mettrons en perspective historique la réforme en cours du dispositif français de normalisation comptable; ce qui nous permettra d'apprécier l'importance du changement qu'elle représente.

Dans une deuxième section, nous exposerons les fondements théoriques de notre démarche interprétative. Ces fondements procèdent des divers courants (économique, sociologique et historique) du néo-institutionnalisme.

Dans une troisième section, nous appliquerons les notions de dépendance de sentier et de mimétisme à l'étude du nouveau CNC et comparerons celui-ci d'une part, au FASB et, d'autre part, à l'AMF, afin de repérer les mimétismes institutionnels qui peuvent expliquer son architecture.

\section{Le « sentier " historique de la normalisation française}

Dans cette section, après avoir rappelé les principales étapes de la normalisation comptable française, nous nous attarderons sur la réforme de son dispositif en 1996-98, réforme que remet en cause le décret du 27 avril 2007.

\subsection{Les principales étapes de la normalisation comptable française}

La réglementation et la normalisation comptables françaises sont anciennes mais ce n'est qu'après la Seconde Guerre Mondiale que la France s'est dotée d'un véritable dispositif de normalisation comptable avec la création en 1946 (décret n 46-619 du 4avril 1947) d’une Commission de Normalisation des Comptabilités, à laquelle succèdera en 1947 (décret n 47188 du 16 janvier) le Conseil Supérieur de la Comptabilité qui sera lui-même remplacé en 1957 ( décret n 57-129 du 7 février) par le (premier) Conseil National de la Comptabilité. 
Ces trois organismes successifs apparaissent rétrospectivement comme les symboles d'une normalisation «à la française » caractérisée par trois traits principaux (Colasse et Standish, 1998, p. 10).

Premièrement, elle est placée sous la tutelle de l'Etat qui en est l'animateur et c'est l’un de ses serviteurs, un haut fonctionnaire, qui présidait jusqu'en 1996 les organismes cités précédemment ${ }^{3}$.

Deuxièmement, elle fait participer à l'élaboration de la norme comptable les diverses parties susceptibles d'être concernées par cette élaboration en ceci que leurs intérêts peuvent être affectées directement ou indirectement par les normes produites. Ces parties sont représentées au sein de l’organisme de normalisation et débattent entre elles. Les décisions, au moins les plus importantes, sont prises par tous les membres du conseil réunis en assemblée plénière. La normalisation «à la française » est une normalisation par la parole, " par la salive » a-t-on dit. D’où une relative lenteur mais qui a sa contrepartie positive en termes d'application.

En effet, troisièmement, en raison du caractère collégial de son élaboration, lequel n’exclut pas évidemment les jeux de pouvoir et son appropriation éventuelle par les parties dominantes, la norme comptable, et en particulier le Plan Comptable Général (PCG), apparaît in fine légitime et n'exige pas un appareil coercitif très puissant pour être mise en œuvre. «Le plan comptable n'est ni loi, ni corps de doctrine officiel; il tire son autorité du consentement et de l'approbation de ses utilisateurs » écrivait de façon sans doute un peu optimiste le président du Conseil Supérieur de la Comptabilité dans son introduction au PCG 1957. Le PCG n'est effectivement que l'annexe d'un arrêté ministériel d'approbation, ce qui le situe assez bas dans la hiérarchie des sources de droit. Toutefois, sous l'influence de l'harmonisation comptable européenne, sera mis en place un embryon de droit comptable, la transposition en droit français des directives européennes d’harmonisation impliquant le vote de lois. D’où la rigidification de la normalisation «à la française » dans une période qui s’ouvre dans les années 1980 et qui exige une forte réactivité normative.

Les années 1980, une fois passée la vague des nationalisations de 1981, seront en effet des années de déréglementation des marchés financiers, de privatisation des entreprises nationalisées et d'ouverture de l'économie française. Dans ce contexte, la normalisation «à la française » est remise en cause, en raison de sa lenteur et de sa rigidité ; d’où la réforme 9698 du Conseil National de la Comptabilité. 


\subsection{La réforme 1996-1998 du dispositif français de normalisation comptable}

Cette réforme s'est traduite par une rénovation du premier CNC et la création d'un nouvel organisme, le Comité de la Réglementation Comptable (CRC). Le décret n 96-746 du 26 août 1996 a redéfini le rôle du CNC, sa composition et son fonctionnement ; et il a créé en son sein un comité d'urgence, ce qui n’existait pas auparavant mais s’imposait désormais dans un contexte économique et financier en pleine évolution.

Comme le premier CNC et les organismes qui avaient précédé celui-ci (Commission de Normalisation des Comptabilités et Conseil Supérieur de la Comptabilité), ce deuxième CNC restait un organisme consultatif placé auprès du ministre chargé de l'économie mais sa mission étaient étendue à l'ensemble des secteurs économiques, y compris les secteurs bancaire et des assurances. Son effectif, dans un souci d'efficacité, passait de 103 membres à 58 membres et la nouvelle répartition de ses membres donnait un poids accru aux «professionnels », représentants des entreprises et de la profession comptable libérale, aux dépens des représentants de l'Etat. Par ailleurs, son président voyait son statut renforcé : ses fonctions étaient désormais « exclusives de toute autre activité, publique ou privée, rémunérée ou non, à l'exception d'activités d'enseignement ou de fonctions exercées au sein d’organismes internationaux $» .{ }^{4}$. Il s'agissait de renforcer la compétence technique du CNC et de le rendre plus efficace et plus réactif.

La création en son sein d’un comité d’urgence répondait aussi directement à l'objectif de réactivité. Ce comité est composé du président, des six vice-présidents, du représentant du garde des sceaux-ministre de la justice, d'un représentant du ministre chargé de l'Economie, d'un représentant du ministre chargé du Budget et du représentant de la Commission des Opérations de Bourse (désormais de l’AMF). Il est saisi par le président ou par le ministre chargé de l'Economie de toute question relative à l'interprétation ou à l'application d'une norme comptable nécessitant un avis urgent. Il doit statuer dans un délai maximum de trois mois à compter de la date de saisine.

Quant au nouveau Comité de la réglementation comptable (CRC), créé par la loi n 98-261 du 6 avril 1998, il a, comme sa désignation l'indique, un rôle règlementaire. Il a pour mission d' "établir des prescriptions générales ou sectorielles » (art. 1 de la loi). Il comprend 15 membres : aux côtés de six représentants ${ }^{5}$ de l’Etat (au sens large), on trouve le président de la Commission des Opérations de Bourse, le président du CNC, le président du Conseil supérieur de l’OEC, le président de la CNCC, trois représentants des entreprises, deux représentants des organisations syndicales représentatives. Il adopte ses règlements au vu des 
recommandations ou après avis du CNC. Ses règlements sont ensuite approuvés par un arrêté conjoint du ministre de l'Economie, du ministre de la Justice et du ministre du Budget qui leur donne force obligatoire.

Avec la création du CRC, le dispositif français de normalisation était désormais composé de deux organismes complémentaires : un organisme chargé d'élaborer les normes, le nouveau $\mathrm{CNC}$, un organisme chargé de les transformer en règlements, le CRC. S’il en corrigeait certains des dysfonctionnements, le nouveau dispositif ne remettait pas fondamentalement en cause la «normalisation à la française ». Son caractère partenarial et collégial était conservé. Par ailleurs, si le poids des entreprises et de la profession ${ }^{6}$ libérale se trouvait incontestablement accru au niveau du CNC, l'Etat gardait implicitement au niveau du CRC une sorte de droit de veto (Colasse et Standish, 1998, p. 21). On attendait avant tout de ce dispositif bicéphale une plus grande efficacité dans un monde économique en pleine transformation mais c'était sans compter avec la montée en puissance de la normalisation comptable internationale et le choix à venir de l'Europe en faveur de cette normalisation ${ }^{7}$.

\subsection{Le nouveau CNC (décret $n^{\circ}$ 2007-629 du 27 avril 2007)}

Si la mission de ce nouveau et troisième CNC n’est guère différente de celle du précédent, sa composition et son fonctionnement le sont assez profondément. Il comprend un collège présidé par l'un de ses membres, des commissions spécialisées, un comité consultatif et une direction générale.

Le collège est l'organe de décision; ses décisions prennent, comme dans le passé, la forme d'avis ou de recommandations.

Il est composé de seize membres dont le mandat est de trois ans (à l'exception de celui du président qui est de six ans) :

- un Conseiller d’Etat désigné par le vice-président du Conseil d'Etat ;

- un Conseiller Maître à la Cour des Comptes désigné par le premier président de la Cour des comptes ;

- un représentant de l'Autorité des Marché Financiers désigné par le Président de l’Autorité des Marchés Financiers ;

- un représentant de la Commission Bancaire désigné par le président de la Commission Bancaire ; et

- neuf personnes désignées, à raison de leur compétence économique et comptable, par le ministre chargé de l'économie ${ }^{8}$, après consultation des organisations représentatives des 
entreprises et des professionnels de la comptabilité, parmi lesquelles le ministre chargé de l’économie désigne le président ;

- un représentant des organisations syndicales représentatives des salariés nommé par le ministre chargé de l'économie après consultation des organisations syndicales.

Ses décisions sont prises à la majorité de ses membres présents ; en cas de partage égal des voix, celle de son président est prépondérante.

Le président du collège est nommé par le ministre chargé de l’économie parmi les neuf personnes désignées à raison de leur compétence économique et sociale. Il est implicitement le président du CNC. Cependant, à la différence du président du CNC de 1996, il ne semble pas tenu de se consacrer exclusivement à sa mission. Il est d'ailleurs à remarquer que l'obligation faite à son président de se consacrer exclusivement au CNC avait été supprimée par un décret du 13 mars 2007 (n²007-336) publié le même jour que l'arrêté nommant M. Jean-François Lepetit à la tête du CNC (1996) et peu de temps donc avant le décret relatif au nouveau CNC. On peut donc penser que le président du collège se bornera dans le futur à animer celui-ci et laissera les activités opérationnelles aux présidents des commissions spécialisées et au directeur général.

Les commissions spécialisées sont constituées par le collège, elles sont chargées de préparer ses projets d'avis. Deux d'entre elles sont permanentes et prévues expressément dans le décret de 2007: la «commission des normes comptables internationales » et la « commission des normes comptables privées »; chacune de ces commissions comprend neuf membres et est présidée et vice-présidée par deux membres du collège désignés par le président.

Le comité consultatif du conseil est composé de vingt-cinq représentants du monde économique et social dont deux représentants des syndicats représentatifs des salariés, nommés pour une durée de trois ans renouvelable par arrêté du ministre chargé de l'économie après avis du président du collège. «Le président du collège réunit au moins une fois l'an le comité consultatif, à qui il présente un rapport d'activité et un programme de travail annuel sur lesquels le comité formule des observations » (art. 5 du décret).

La direction générale est chargée de la gestion administrative du conseil, de la préparation des avis et des recommandations du collège et du suivi des travaux techniques. Son responsable, le directeur général, est nommé par arrêté du ministre chargé de l'économie, après avis du président du collège ; il assiste aux réunions des formations du conseil. 
Ainsi que son principal promoteur, M. Jean-François Lepetit, l’a annoncé, cette nouvelle réforme du CNC n’est qu'une première étape vers la création d'une Autorité des Normes Comptables (ANC) françaises. Fin 2007, une loi devrait en effet confier ses prérogatives règlementaires au CNC rénové et abroger la loi de 1998 qui lui a donné naissance. Comme les règlements du CRC, ceux du CNC seraient homologués par arrêté et acquerraient ainsi force obligatoire. Cette loi ferait du CNC la seule autorité compétente en matière de normes comptables privées.

Il apparaît que ce troisième CNC est fort différent de ses prédécesseurs et rompt avec la normalisation comptable « à la française » et, en particulier, avec son caractère partenarial. Les différentes parties prenantes à la chose comptable sont désormais cantonnées dans le comité consultatif qui apparaît comme le fossile de l'assemblée plénière des premiers et deuxième CNC. Alors que cette assemblée avait un rôle décisionnel, ce comité, comme le qualificatif l'indique, n'est que consultatif. C'est le collège qui décide et les lieux d'élaboration des normes sont les commissions spécialisées, lesquelles sont composées d'experts et non pas de représentants. On peut donc s'attendre à une " professionnalisation » de la normalisation comptable, a l'instar de ce qui se passe dans les pays anglo-saxons et au plan international. Une telle professionnalisation se traduit le plus souvent par un recours important à des experts fournis et rémunérés par les grands cabinets ou les grandes entreprises et, de fait, à une mainmise de ceux-ci et de celles-ci sur la normalisation. Si l'on considère par exemple la composition de la commission des normes comptables internationales décidée par le président du CNC à l’occasion de la première réunion du Collège du 19 décembre 2007, on remarque que cette commission de neuf membres est composée de quatre personnalités appartenant à de grands cabinets (Ernst, Deloitte, KPMG et Mazars), et de quatre personnalités appartenant à des entreprises du CAC 40 (AXA, BNP, Peugeot, Suez) ; quant au président (M. Gérard Gil) et au vice-président ( $M$. Dominique Thouvenin) de cette commission, ils appartiennent respectivement à la BNP et à Ernst.

Tout ceci conduit à s’interroger sur le sens de cette réforme et la nature du nouveau CNC et de la future ANC. Pour tenter de répondre à cette interrogation, nous emprunterons à l'analyse institutionnelle.

\section{L'analyse des institutions de régulation comptable}


La régulation comptable recouvre la production, la mise en œuvre et le contrôle de l’application des normes comptables. Elle se déploie dans un espace géopolitique donné, qui peut être national ou international. Dans cet espace, la régulation comptable est centrée sur les normes et s’appuie sur un dispositif comportant généralement les éléments suivants : un (ou des) organisme(s) chargé(s) de leur élaboration, une entité fédérant la profession comptable qui aura à mettre en œuvre les normes, une autre encadrant celle d'auditeur qui devra en vérifier l'application et enfin, un organisme supervisant de manière indépendante l'exercice de la profession d'auditeur.

Dans cet article, nous nous intéressons à un élément particulier du dispositif : les organismes chargés de la production des normes comptables. Nous tenterons de montrer dans un premier temps que ces organismes peuvent être considérés comme des institutions. Nous proposerons ensuite un cadre théorique pour les analyser d'un point de vue statique et dynamique.

\subsection{Les organismes de normalisation comptable comme institutions}

Qu'est-ce qu'une institution ? Pour le chercheur en sciences de gestion, répondre à cette question est une entreprise délicate. Les références abondent en effet car le concept d'institution est largement mobilisé par les diverses sciences sociales (au premier rang desquelles la sociologie mais également l'économie, le droit et les sciences politiques). Cette variété de références, si elle enrichit la réflexion sur la notion d'institution en accroît simultanément le caractère polysémique, chaque discipline abordant le concept du point de vue particulier qui est le sien.

Durkheim (1993) établit une filiation directe entre le concept d'institution et la sociologie en tant que science sociale. Dans la préface de la seconde édition (1895) des Règles de la méthode sociologique, il indique : «On peut appeler institution toutes les croyances et tous les modes de conduite institués par la collectivité ». Il précise ensuite : "La sociologie est la science des institutions, de leur genèse et de leur fonctionnement ». Par rapport au fait social, autre concept clé de la pensée de Durkheim, l’institution présente la caractéristique d’être dotée d'une certaine stabilité dans le temps.

Pour la sociologie néo-institutionnelle (Meyer et Rowan, 1977 ; DiMaggio et Powell, 1983), les institutions sont avant tout des normes. Leur dimension cognitive est mise en avant : habitudes de faire et de penser socialement validées sont intériorisées par les membres de la société au point d'apparaître non plus comme une construction sociale mais comme quelque chose de naturel (taken for granted). L'approche sociologique des institutions en 
souligne le caractère dual, à la fois contraignantes (car elles imposent une discipline comportementale) et habilitantes (dans la mesure où elles permettent d'anticiper le comportement des autres membres de la société).

Les économistes reprennent à leur compte l’idée que les institutions s’apparentent aux règles du jeu (par exemple North, 1990), en y incluant à la fois les règles formelles (lois, procédures) et les règles informelles (coutume, usages, habitudes). Pour North, institutions et organisations constituent deux objets distincts : les premières sont les règles du jeu et les secondes sont les joueurs qui les appliquent. Toutefois, l'économie néo-institutionnelle privilégie un point de vue instrumental, c'est-à-dire que les auteurs se rattachant à ce courant s'intéressent aux institutions du seul point de vue de leur impact sur l'efficience, par exemple à travers leur capacité à diminuer les coûts de transaction (Williamson, 1985).

Une autre ligne de fracture dans la manière d'appréhender les institutions sépare les approches centrées sur le résultat du processus de construction des institutions (ce qui est institué) et celles qui mettent l'accent sur l'analyse du processus lui-même (le fait d'instituer ou phénomène d'institutionnalisation). A cet égard, l'un des reproches adressés à la sociologie néo-institutionnelle ${ }^{9}$ est de passer sous silence la façon dont émergent les institutions. L'analyse est centrée sur les effets produits par les institutions existantes plutôt que sur leur mode de génération. A contrario, certaines sciences sociales comme le droit (notamment le droit public) et les sciences politiques s'intéressent à la genèse des institutions. Leur apport est particulièrement important pour notre propos.

Hauriou, qui fut professeur de droit administratif et doyen de la faculté de droit de Toulouse (1906-1926), est l'auteur d'une théorie institutionnelle dans laquelle il développe une conception des institutions indissociable de la manière dont elles sont construites. Pour Hauriou (1925), une institution naît en effet de l'articulation de trois éléments : une idée d'œuvre (on dirait aujourd'hui une idée directrice), un pouvoir de gouvernement organisé et « des manifestations de communion », c'est-à-dire une acceptation collective de la concrétisation de l'idée d'œuvre ${ }^{10}$. Hauriou appelle intériorisation le mouvement par lequel ces trois éléments s'enchaînent, l'assentiment majoritaire succédant à l'intuition minoritaire. L’intérêt de cette approche réside dans son caractère éminemment dynamique. Hauriou considère également deux types d'institutions, les institutions-personnes ou corps et les institutions-choses (Millard, 1995). Les institutions-personnes constituent des collectivités humaines particulières au service de l'accomplissement d'une idée d'œuvre (l'école, l'entreprise, l'église) tandis que les institutions-choses (le droit de propriété par exemple) seraient autonomes et pourraient vivre sans le support d’un corps spécifique. 
Cette opposition entre institutions-personnes et institutions-choses recoupe la distinction établie par Bensedrine et Demil (1998) entre institutions matérielles et immatérielles. Ainsi que le remarquent ces auteurs, l'approche matérialiste des institutions est à bien des égards complémentaire de celle qui place la règle au centre de l'analyse. En effet, «les institutions immatérielles (cognitives, coercitives, normatives) sont souvent associées à des organisations qui les soutiennent ». Si nous retenons cette perspective duale pour aborder l'étude du système de régulation comptable, il apparaît alors que les divers éléments qui le composent revêtent tous la nature d'institutions mais que celles-ci prennent soit la forme d'institutions immatérielles (les normes comptables, cœur du dispositif), soit celle d'institutions matérielles (l'organisme de normalisation comptable, les associations professionnelles de comptables et d'auditeurs, l'organisme indépendant chargé du contrôle de la profession d'auditeur) dont la raison d’être est directement liée aux normes.

Les dispositifs de normalisation comptable sont donc des institutions au même titre que les normes qu'ils ont pour fonction de produire. Ces institutions se matérialisent sous la forme d'organisations dont la nature (publique ou privée), la taille, la structure et la composition sont susceptibles de varier dans le temps comme dans l'espace. Il est néanmoins possible de repérer en statique un certain nombre de formes-types.

\subsection{Les formes-types des organismes de normalisation comptable : à la recherche d'une double légitimité}

Si l'on entend par formes-types ou, pour employer un vocabulaire wébérien, idéaux-types de la normalisation comptable, les formes organisationnelles qui reposent sur un seul acteur social, l'observation historique et géographique permet d'en distinguer trois (Colasse, 2005) qui reposent respectivement sur l'Etat, la profession comptable ou un organisme indépendant. A travers ces forme-types, il est en particulier possible de mettre en évidence la double contrainte de légitimité, technique et politique, qui pèse sur une institution de normalisation comptable.

La normalisation par l'Etat ou sous la tutelle de l'Etat, entendu au sens large comme incluant le législatif, l'exécutif et le judiciaire, confère évidemment aux normes émises une forte légitimité, puisqu'elle procède du représentant de l'intérêt commun, et une grande effectivité, puisqu'elles sont soutenues par un appareil coercitif puissant. Par contre, les normes émises peuvent manquer de qualité technique. En effet, dans de nombreux pays, l’Etat ne dispose pas de fonctionnaires suffisamment compétents pour produire des normes dans un domaine aussi technique que le domaine comptable. Par ailleurs, l'appareil d'Etat est un 
appareil lourd qui manque souvent de réactivité dans le traitement d'une matière très évolutive et de problèmes qui appellent des solutions en urgence. C'est ce que l'on a pu observer en France dans les années 1990 quand le CNC, organisme sous la tutelle de l’Etat, n’a pu répondre aux nouvelles demandes de normes liées au développement accéléré des marchés financiers et à l'apparition de nombreux nouveaux produits financiers.

La normalisation par la profession, plus précisément par la profession libérale, que l’on désigne encore sous le vocable d'endo-régulation, confère a priori une grande légitimité technique aux normes émises ; les membres de la profession libérale étant considérés, au moins dans les pays développés, comme les mieux informés des problèmes et les plus compétents pour les traiter. Par contre, si elle apparaît donc, malgré certaines réserves, comme très légitime au plan technique, elle le semble beaucoup moins au plan politique. En effet, la comptabilité et les informations qu'elle produit concernent une pluralité d'acteurs économiques et sociaux qui sont autant de parties prenantes potentielles à sa normalisation. La profession comptable libérale n'est donc que l'une des parties prenantes à la chose comptable et les autres peuvent à juste titre craindre qu'en tant que régulateur elle manque de neutralité et n’oriente les normes dans le sens de ses intérêts corporatistes ; par exemple en élaborant des normes sophistiquées qui nécessitent son intervention et contribuent ainsi à augmenter la demande de prestations comptables et d'audit qui lui est adressée. Par ailleurs, elle n'est pas homogène, et les dissensions qui peuvent surgir en son sein (par exemple, entre petits et grands cabinets ou entre cabinets nationaux et cabinets internationaux) peuvent provoquer des blocages et avoir des conséquences néfastes sur le processus de régulation luimême. Ainsi, les cabinets franco-français et les cabinets internationaux n’accueillent pas de la même façon les normes internationales ; ne serait-ce que parce que la généralisation de cellesci donnera vraisemblablement un avantage compétitif à ces derniers et induira une restructuration du marché de la prestation comptable et d'audit aux dépens des cabinets nationaux.

Les limites respectives des deux idéaux-types précédents amènent au troisième : la régulation par un organisme voulu indépendant à la fois de l’Etat et de la profession. On peut concevoir un régulateur indépendant de deux façons assez différentes et l'on peut parler de régulateurs indépendants du « premier type » et du « deuxième type » (Colasse, 2005).

Premièrement, dans une conception représentative, comme un organisme partenarial composé des représentants des différentes parties, y compris l’Etat et la profession, concernées par l'information comptable ; ce qui pose évidemment le problème complexe du choix des acteurs économiques et sociaux qui pourront y être représentés et de la place qu’il 
convient d'accorder à chacun d'entre eux. Les premier et deuxième CNC sont assez proches de cette forme-type : ils sont certes sous la tutelle de l'Etat mais leurs membres, dans leurs grande majorité, sont des représentants des diverses parties prenantes.

Deuxièmement, dans une conception technocratique, comme un organisme composé de spécialistes chevronnés (d' " experts ») censés indépendants; mais reste, dans cette conception, à définir ce qu'est un individu indépendant et à trouver des modes de désignation et de rémunération qui préservent son indépendance. Le FASB ${ }^{11}$ et l’IASB illustrent cette conception d'un organisme indépendant. Ce qui ne signifie pas qu'ils le soient réellement. On a souvent dénoncé aux Etats-Unis la mainmise des grands cabinets sur les travaux du FASB (voir par exemple Briloff, 1982)

Selon qu'il est du premier ou du deuxième type, le régulateur indépendant risque de se heurter dans sa tâche à des difficultés différentes.

Composé de représentants des différentes parties prenantes qui débattent ensemble, sa légitimité politique est a priori forte. Par contre, parce qu'il lui faut constamment chercher des compromis en son sein, ses normes peuvent s'avérer peu satisfaisantes d'un point de vue technique et amoindrir sa légitimité; de plus, son caractère collégial fait qu’il réagit peu rapidement au besoin de norme.

Composé de spécialistes indépendants, sa légitimité technique est a priori forte. Par contre, dans la mesure où ces spécialistes ne représentent en principe aucune partie prenante, sa légitimité politique est plus faible, ce qui peut constituer un obstacle à la bonne acceptation de ses normes par les assujettis. Il lui faut donc agir selon des procédures aussi transparentes que possible qui permettent la discussion de ses travaux et disposer des soutiens publics ou para-publics susceptibles de lui donner la légitimité politique qui lui manque.

A la suite de l'examen de ces formes-types de la régulation comptable, il apparaît que tout régulateur comptable est confronté à un problème plus ou moins aigu de légitimité, soit technique, soit politique. Disons que le régulateur public se trouve plutôt exposé à un problème de légitimité technique et ce d'autant plus que les problèmes comptables deviennent de plus en plus complexes, tandis que le régulateur privé est plutôt exposé à un problème de légitimité politique. Ceci peut conduire à penser (Colasse, 2005) qu'une bonne régulation dans un domaine aussi complexe que l'est le domaine comptable ne peut reposer que sur une collaboration entre le privé et le public, l'un apportant sa légitimité technique à l'élaboration des normes, l'autre sa légitimité politique à leur mise en application.

Il convient également de noter que ces formes institutionnelles ne produisent pas le même «droit » comptable. Le droit produit par l’Etat ou un organisme qui en dépend 
étroitement est, si l'on reprend le vocabulaire de Delmas-Marty (2004), plus dur (hard) que celui élaboré par un organisme professionnel. La collaboration ou le partenariat entre public et privé produit une combinaison de droit dur (hard law) et de droit mou (soft law).

\subsection{La dynamique des dispositifs de normalisation comptable : un triple cadre d'analyse}

Comme toutes les institutions, les dispositifs de régulation comptable sont appelés à évoluer au cours du temps. Ceci vaut tout d'abord pour les normes comptables: d'une part, les référentiels nationaux ont dû s’adapter aux modifications de l'environnement économique (par exemple, l'apparition de nouveaux actifs financiers), d'autre part, ils ont subi l'influence du mouvement d'harmonisation qui s’opère à l'échelle internationale. L'évolution des dispositifs de régulation comptable concerne également les institutions matérielles adossées au référentiel normatif et notamment l'institution dont la mission est de produire ce référentiel. Nous proposons de recourir à un triple cadre d'analyse pour interpréter l'évolution d'une institution de normalisation comptable telle que le CNC. Empruntant aux travaux des chercheurs en sciences politiques, nous distinguons trois courants théoriques au sein de la perspective néo-institutionnelle. L'un relève d'une approche économique, l'autre d'une approche sociologique et le dernier d'une approche historique.

\subsubsection{La dynamique institutionnelle dans la nouvelle économie institutionnelle}

La nouvelle économie institutionnelle s'est développée dans le prolongement des travaux de Coase (1937) sur la nature de la firme, autour du concept de coûts de transaction. Son objet est la comparaison, sur la base des coûts de transaction, des diverses alternatives institutionnelles au fonctionnement constaté des marchés. S’agissant plus particulièrement de l'environnement institutionnel, l’idée centrale développée par North (1990) est que la croissance économique est conditionnée par la capacité des institutions à faciliter et à sécuriser les transactions. Tous les dispositifs institutionnels ne se valent donc pas, certains sont plus efficients que d'autres : ceux qui permettent une réduction plus importante des coûts de transaction.

Appliqué à la gouvernance d'entreprise, ce cadre théorique a suscité des développements connus sous le nom de théorie juridico-financière (Law and Finance). Selon LaPorta et al. (1998), tous les systèmes nationaux de gouvernance ne se valent pas. Certains sont plus efficients que d'autres parce qu'ils offrent aux droits des actionnaires minoritaires une protection juridique de qualité, condition nécessaire du développement des marchés financiers. De cet état de fait résulte une concurrence normative (regulatory competition) 
poussant les pays dont les systèmes de gouvernance sont les moins performants à les modifier en adoptant les institutions (et notamment les règles de droit des affaires) ayant fait leurs preuves ailleurs. Selon cette perspective, c'est la recherche d'une efficience économique accrue qui motive le changement institutionnel et cette dynamique a pour effet de faire converger les systèmes nationaux de gouvernance.

En tant que mécanisme de gouvernance d'entreprise, le dispositif de régulation comptable (et en particulier l'institution de normalisation) peuvent se voir appliquer le raisonnement précédent. Si certains référentiels normatifs dominent les autres au plan de l'efficience parce qu'ils procurent aux actionnaires une information financière de meilleure qualité sur les entreprises, ils devraient logiquement s'imposer et remplacer progressivement les référentiels de moindre qualité. L’harmonisation internationale des normes comptables s'interprète dans cette perspective comme un mouvement de convergence vers les normes les plus efficientes. De même, la forme organisationnelle de l'institution de normalisation n'est pas neutre. Selon la façon dont cette institution fonctionne, les normes qu'elle produit répondront plus ou moins fidèlement aux attentes des actionnaires, critère qui détermine leur degré d'efficience selon la perspective économique. Là encore, une logique darwinienne devrait conduire à l'élimination des formes organisationnelles les moins efficientes.

Du point de vue de l'analyse dynamique des institutions dans une perspective comparative, l'intérêt des théories économiques néo-institutionnelles est donc de fournir un critère de comparaison, dans le temps et/ou dans l'espace, de dispositifs institutionnels remplissant une fonction identique. Un tel critère possède un potentiel explicatif tout à la fois de l'évolution dans le temps de ces dispositifs et de la logique du processus de diffusion internationale des institutions (Pochet, 2007).

\subsubsection{La dynamique institutionnelle dans la sociologie néo-institutionnelle}

Alors que l'économie néo-institutionnelle focalise son attention sur les différences (en termes de coûts de transaction) entre institutions alternatives, la sociologie néo-institutionnelle a pour projet essentiel d'expliquer la propension à se ressembler des organisations œuvrant dans un même champ. L'accent est donc mis ici sur la similitude formelle des organisations plongées dans le même environnement institutionnel, résultat d'un processus appelé isomorphisme (DiMaggio et Powell, 1991). Deux types d'isomorphisme sont dentifiés : un isomorphisme dit concurrentiel, qui résulte du mécanisme de sélection naturelle décrit par la nouvelle économie institutionnelle et un isomorphisme institutionnel.

Le concept d'isomorphisme institutionnel repose sur l’idée que «les organisations sont en concurrence, non seulement pour les ressources et les clients, mais aussi pour le 
pouvoir politique et la légitimité institutionnelle » (DiMaggio et Powell, 1991). Dans cette perspective, la quête de similitude par les organisations obéit à une logique de légitimité et non d'efficacité. La similarité facilite en effet l'acquisition d'une légitimité par les organisations dans la mesure où les éléments copiés (structures formelles, technologies, outils de gestion,...) sont ceux qui ont été préalablement validés socialement. DiMaggio et Powell identifient trois moteurs de changement institutionnel isomorphique: coercition, normalisation et mimétisme.

Les sciences politiques ont fait du cadre sociologique néo-institutionnel un usage fréquent pour rendre compte des processus d'isomorphisme institutionnel dans le domaine des politiques publiques. Si les organisations deviennent similaires, c'est parce qu'elles en viennent à calquer leurs structures et leurs pratiques sur celles d'autres organisations, conférant en cela le caractère d'institutions à ces structures et pratiques. Par analogie, les chercheurs en sciences politiques ont suggéré que les responsables des politiques publiques tendaient à imiter celles des pays les plus performants et avec elles, les institutions qui portent ces politiques, afin de gagner en légitimité auprès des électeurs : dans ce contexte, «le design institutionnel relève typiquement de la copie et il est fréquemment revendiqué comme tel » (Offe, 1996). Plusieurs études empiriques dans ce champ de recherche tendent à confirmer tant les phénomènes de mimétisme que leur interprétation en termes de quête de légitimité. On peut ainsi mentionner les travaux de Radaelli (2000) concernant les politiques monétaire et fiscale de l’Union Européenne ou ceux que Schneider (1998) a consacrés aux politiques de régulation du secteur des télécommunications en Europe.

Dans cette perspective, la logique sous-tendant l'évolution d'une institution de normalisation comptable serait à chercher dans l'imitation de formes organisationnelles existant dans d'autres pays et ayant reçu une validation à l'échelle internationale. L'imitation d'un modèle perçu comme performant conférerait dans ce cas une légitimité à la réforme institutionnelle portée par les responsables politiques du pays qui l'initierait. Il est important de souligner que l'imitation d'un modèle socialement validé dans un autre contexte national peut se révéler dysfonctionnelle dans le pays imitateur sans pour autant affecter la légitimité de l'institution.

\subsubsection{La dynamique institutionnelle dans le néo-institutionnalisme historique}

Si l'économie et la sociologie néo-institutionnelles constituent des cadres théoriques d'analyse familiers des chercheurs en sciences de gestion, il n'en est pas de même pour le néo-institutionnalisme historique. Ceci s’explique sans doute, partiellement au moins, par le fait que l'approche historique des institutions a eu pour terrain d'analyse privilégié l'Etat, 
sous ses diverses formes institutionnelles. Ce sont donc essentiellement les sciences politiques qui ont fait usage de ce cadre théorique pour tenter d'expliquer un certain nombre d'observations, notamment l'existence de trajectoires nationales diverses. Le propos d'une large part de cette littérature est ainsi de nature comparative et porte sur la comparaison internationale des politiques publiques et des institutions qui les sous-tendent. Si ce cadre théorique nous paraît a priori pertinent pour notre objet analyse, c’est évidemment en raison de l'influence qu'exerce l'Etat sur le CNC à travers son rôle de tutelle.

L’un des apports conceptuels essentiels du néo-institutionnalisme historique réside dans la notion de dépendance de sentier (path dependence). A travers ce concept, l'accent est mis sur le caractère relativement stable des institutions et sur la capacité de ces dernières à orienter la trajectoire économique d'un pays le long de «sentiers » particuliers. De ce fait, soumis à des forces identiques, deux pays pourront emprunter des trajectoires sensiblement différentes. La dépendance de sentier implique que l'environnement institutionnel hérité du passé va façonner les choix effectués par l'Etat à un moment donné en matière de politiques publiques (Weir et Skocpol, 1985). Une telle conception ne s'oppose pas radicalement à l'idée que la dynamique institutionnelle est sous-tendue par un principe d'efficience. Elle soutient seulement que cette quête d'efficience est contrainte par l'histoire. Ceci permet notamment d'expliquer pourquoi des institutions inefficientes persistent. Avec leur analyse des «variétés du capitalisme, Hall et Soskice (2001) montrent qu’à l'échelle macroéconomique, certaines institutions et organisations sont complémentaires et se renforcent mutuellement au cours du temps. Ce processus de coévolution favorisant certaines configurations institutionnelles plutôt que d'autres permet d'expliquer les trajectoires distinctes empruntées par des pays soumis à des pressions environnementales similaires.

Selon cette perspective, le changement institutionnel n'est pas graduel. A des périodes de forte stabilité institutionnelle vont succéder des phases de crise marquées par un changement substantiel des institutions et l'orientation sur une nouvelle trajectoire.

Un tel cadre théorique peut permettre d'expliquer la persistance de certains éléments formels hérités du passé au sein d’une institution dont la forme a évolué au cours du temps comme ce fut le cas pour le CNC. Il peut également suggérer des éléments d'explication à la diversité des formes institutionnelles revêtues par les organismes de normalisation comptable de par le monde.

Le tableau suivant présente une vue synthétique des apports de notre triple cadre théorique à l'analyse de la dynamique institutionnelle. Une proposition concernant l'évolution attendue du CNC selon chacune des trois perspectives est formulée. 
Tableau 1 : Trois cadres théoriques pour l'analyse de la dynamique institutionnelle

\begin{tabular}{|c|c|c|c|}
\hline Cadres théoriques & $\begin{array}{c}\text { Nouvelle économie } \\
\text { institutionnelle }\end{array}$ & $\begin{array}{c}\text { Sociologie néo- } \\
\text { institutionnelle }\end{array}$ & $\begin{array}{c}\text { Néo- } \\
\text { institutionnalisme } \\
\text { historique }\end{array}$ \\
\hline Concept central & Coûts de transaction & Isomorphisme & $\begin{array}{c}\text { Dépendance de } \\
\text { sentier }\end{array}$ \\
\hline $\begin{array}{c}\text { Principe sous-tendant } \\
\text { la dynamique } \\
\text { institutionnelle }\end{array}$ & Efficience & Légitimité & Efficience contrainte \\
\hline $\begin{array}{c}\text { Convergence des } \\
\text { formes }\end{array}$ & $\begin{array}{c}\text { Oui, par le biais d'un } \\
\text { processus de } \\
\text { sélection naturelle }\end{array}$ & $\begin{array}{c}\text { Oui, sous l'effet de } \\
\text { l'isomorphisme } \\
\text { institutionnel } \\
\text { (coercitif, normatif, } \\
\text { mimétique) }\end{array}$ & $\begin{array}{c}\text { Non, maintien de } \\
\text { différences en raison } \\
\text { du poids de l'histoire }\end{array}$ \\
\hline $\begin{array}{c}\text { Proposition } \\
\text { concernant la } \\
\text { dynamique du CNC }\end{array}$ & $\begin{array}{c}\text { La nouvelle } \\
\text { organisation du CNC } \\
\text { devrait lui permettre } \\
\text { efcroître son }\end{array}$ & $\begin{array}{c}\text { La nouvelle } \\
\text { organisation du CNC } \\
\text { devrait ressembler à } \\
\text { celle des } \\
\text { normalisateurs } \\
\text { nationaux jouissant } \\
\text { d'une forte légitimité }\end{array}$ & $\begin{array}{c}\text { La nouvelle } \\
\text { devanisation du CNC } \\
\text { d'accroître son } \\
\text { efficience tout en } \\
\text { conservant des traces } \\
\text { de son passé }\end{array}$ \\
\hline
\end{tabular}

\section{Application à la compréhension de la genèse du nouveau CNC}

Le nouveau CNC se trouve, semble-t-il, et c'est l'hypothèse que nous allons tester dans cette section, à la croisée de deux isomorphismes : un isomorphisme qui le tire vers les fondations à l'anglo-saxonne et un autre qui le tire vers les autorités administratives indépendantes à la française. Cette hypothèse relève d'une dynamique institutionnelle susceptible d'être éclairée par le cadre théorique précédemment tracé. Si isomorphisme il y a, celui-ci peut en effet relever d'une quête d'efficacité ou de légitimité. Il peut aussi être contraint par l'histoire de l'institution considérée. Pour tester cette hypothèse, nous allons comparer le nouveau CNC d'une part, avec le Financial Accounting Standards Board (FASB) et, d'autre part, avec l'Autorité (française) des Marchés Financiers (AMF). Cette double comparaison procède pour l'essentiel d'une étude documentaire.

\subsection{Une comparaison entre le FASB et le nouveau CNC}


La plupart des institutions de régulation comptable anglo-saxonnes sont plus ou moins calquées sur le modèle du FASB américain. Outre qu'il vient du pays dominant, ce modèle véhicule les symboles de la compétence et de l'indépendance et l’on comprend qu'il soit très attractif et provoque le mimétisme. Il a été en particulier adopté par la plupart des pays anglosaxons pour leur normalisateur national ; et c'est aussi le modèle qui, très clairement, a inspiré en 2001 la réforme de structure du normalisateur international (Colasse, 2004) : l'ancien IASC devenant une fondation et cédant ses activités de normalisation au nouvel IASB

Le FASB est la composante opérationnelle d'un dispositif de normalisation chapeauté par une fondation, la Financial Accounting Foundation (FAF).

Mise en place en 1972, la FAF est l'une de ces nombreuse fondations privées américaines qui assument des missions d’intérêt général. En l’occurrence, elle s’est donnée pour objet principal l'élaboration et le perfectionnement des normes de comptabilité et d'information financière. Elle poursuit cet objet en finançant, en nommant les membres et en supervisant les activités non seulement du FASB mais aussi du Governmental Accounting Standards Board (GASB), ainsi que leurs Advisory Councils respectifs, le Financial Accounting Standards Advisory Council (FASAC) et le Governmental Accounting Standards Advisory Council (GASAC).

Sont membres de la fondation et représentés dans son conseil (les trustees) : l'American Accounting Association, l'American Institute of Certified Public Accountants, le CFA Institute, la Financial Executives International, la Government Finance Officers Association, l'Institute of Management Accountants, la National Association of State Auditors, Comptrollers and Treasurers, la Securities Industry and Financial Markets Association. Les trustees sont au nombre de dix-huit; ils sont désignés pour trois ans et peuvent être renouvelés une fois.

Au cœur du dispositif, le FASB. Composé de sept membres choisis exclusivement sur des critères d'indépendance et de compétence, il est un organisme indépendant du «deuxième type ». Ses membres, des spécialistes chevronnés, doivent se consacrer à plein temps à leur activité de normalisateur et ne peuvent avoir d'autres activités.

Le FASAC est composé de plus de trente membres désignés pour un mandat de trois ans renouvelable. Ses membres représentent les préparateurs, les auditeurs et les utilisateurs de l'information comptable. Il a principalement une mission de conseil auprès du FASB : il aide celui-ci à définir son programme de travail et ses priorités, et éventuellement à constituer ses groupes d’études. Le GASAC, créé en 1984, joue un rôle similaire auprès du GASB. 
Si l'on compare le nouveau CNC au dispositif américain de normalisation tel que l'on vient de le décrire sommairement, deux manifestations de mimétisme apparaissent mais qui doivent être immédiatement fortement nuancées.

Tout d'abord, le collège du nouveau CNC s'apparente, mais s’apparente seulement, avec le FASB.

Ils ont certes la même mission, produire des normes, mais, dans le cas du CNC, il s'agit d'une mission statutaire tandis que, dans le cas du FASB, il s'agit d'une mission déléguée ; aux Etats-Unis, c’est en effet la SEC qui détient le pouvoir de normaliser et, d'une certaine façon, le FASB doit lui rendre des comptes quant à la façon dont il assume sa délégation.

L'un et l'autre ont sensiblement le même nombre de membres « opérationnels » et ceux-ci, sept et neuf, sont choisis sur des critères de compétence.

Mais il semble bien que le mimétisme entre le collège du nouveau CNC et le FASB s’arrête là. Plusieurs différences importantes apparaissent immédiatement.

Le collège du CNC, s’il comprend (article 3-II du décret) neuf personnes désignées pour leur compétence comprend également six personnes représentant des institutions étatiques (Conseil d'Etat, Cour de cassation, Cour des comptes) ou paraétatiques (AMF, Commission bancaire, Autorité de contrôle de l'assurance et des mutuelles), ainsi qu'un représentant des organisations syndicales. Il est donc composé à la fois de représentants de l'Etat (au sens large) et d'experts; il ne peut donc être considéré comme un organisme indépendant du « deuxième type ».

Par ailleurs, à l'exception de son président, à la différence des membres du FASB, les membres « opérationnels » du collège n'exercent pas leurs fonctions à plein temps et pourront difficilement, si grande que puisse être leur compétence, mobiliser complètement celle-ci au service de la normalisation.

L’organe du nouveau CNC qui l'apparente le plus au normalisateur américain est son comité consultatif, lequel peut être rapproché du Financial Standards Advisory Council (FASAC). Ils ont en effet une composition et un rôle voisins. Pour autant, peut-on parler de mimétisme ? Sans doute pas si, dans la perspective du néo-institutionnalisme historique, l’on considère le comité consultatif du nouveau CNC comme un vestige de l'assemblée plénière de l'ancien CNC et d'une conception partenariale et collégiale de la normalisation comptable. Ce comité consultatif apparaît en effet davantage comme un «héritage » du passé de la normalisation française que comme une imitation du FASAC ; son rôle apparaît en particulier très limité au regard du rôle de ce dernier. Le nouveau CNC, ainsi que nous l'avons vu en 
première partie, est dépendant d'un sentier historique (path dependency), celui de la normalisation française depuis l'après-guerre.

Enfin, ce qui distingue fondamentalement le nouveau CNC du FASB et met à mal l'hypothèse du mimétisme, c'est le mode de désignation de ses membres et son financement. Les neuf membres «opérationnels » (sur seize) du Collège sont désignés par le ministre chargé de l'économie «à raison de leur compétence économique et comptable » mais sans que leur soient imposées de véritables contraintes d’indépendance, comme les anglo-saxons savent les formuler; l'article 4 du décret se borne en effet à exiger qu'ils fournissent au président et à leurs pairs (les autres membres du collège) un certain nombre d'informations sur leurs fonctions et responsabilités passées, présentes et futures ${ }^{1213}$. Quant au financement du nouveau CNC et à la rémunération du Président et des membres du Collège, il semble, selon les termes de l'article 3-IV du décret, qu'ils doivent être assumés par l’Etat : «Le régime indemnitaire du président et des membres du collège est déterminé par un arrêté du ministre de l'économie »; toutefois (voir plus loin,§ 3.3.2.), le rapport Lepetit propose un financement tripartite de la future ANC. Comme nous l'avons indiqué plus haut, le FASB est quant à lui financé par la FAF dont le mode de financement a été réformé en profondeur par la loi Sarbanes-Oxley (section 109) afin d'en accroître l'indépendance à l'égard de la profession comptable. En complément des revenus qui lui sont procurés par la vente de ses publications $^{14}$, et au lieu des cotisations et des contributions volontaires versées par les membres de la FAF, ce sont aujourd'hui des taxes acquittées par les entreprises cotées (accounting fees) qui financent le FASB.

On remarque encore que le dispositif de normalisation américain comprend un organe compétent pour les normes publiques, le GASB. Or le rapport Lepetit propose que la future ANC comporte « un pôle « normes comptables publiques » » qui serait chargé de préparer les travaux de l'actuel Comité de comptabilité publique et intègrerait l'actuelle Mission des normes comptables publiques ; une telle proposition va dans les sens du processus de mise en convergence entre comptabilité publique et comptabilité privée et entre normes publics et normes privées initié par la LOLF. Si ce pôle était créé, selon des modalités à définir par la future loi, cette création pourrait apparaître comme l'expression d'un mimétisme par rapport au dispositif américain.

\subsection{Une comparaison entre l'AMF et le nouveau CNC}

Certes, l'AMF et le CNC diffèrent d'emblée par la nature de la mission qui leur est confiée : réguler les marchés financiers pour la première, produire les normes comptables pour le 
second. Deux faits nous suggèrent pourtant de comparer le nouveau CNC à l'AMF : d'une part, le fait que soit utilisée l'expression d' « autorité des normes comptables » pour désigner le futur organisme français de normalisation, expression qui fait écho à l'expression « autorité des marchés financiers »; d’autre part, le fait que le principal artisan de la réforme, devenu le président du CNC, Monsieur Jean-François Lepetit, ait exercé les fonctions de président de la Commission des Opérations de Bourse (COB) et participé à l’intégration de celle-ci dans l'AMF.

Si les missions respectives de l'AMF et du CNC diffèrent par leur périmètre d'exercice (les marchés financiers et leurs acteurs d'une part, l'ensemble des entreprises assujetties à la tenue d'une comptabilité d'autre part), la nature de la mission qu'elles poursuivent, la régulation d'entités économiques, constitue un point commun aux deux institutions. A l'intersection des entités régulées par l'AMF et le CNC, on trouve les entreprises faisant appel public à l'épargne. Les missions des deux institutions sont, à l'évidence, complémentaires et d'une certaine manière, on peut considérer que celle du CNC est subordonnée à celle de l'AMF. En effet, une information financière de qualité est essentielle au bon fonctionnement des marchés financiers et la qualité des normes comptables, si elle ne constitue pas une condition suffisante de la qualité de l'information transmise au marché, en constitue certainement une condition nécessaire.

Le contrôle vertical exercé dans certains pays par le régulateur des marchés financiers sur le normalisateur comptable constitue sans doute la meilleure preuve de la complémentarité des missions poursuivies par les deux régulateurs. Ainsi, aux Etats-Unis, le Securities Exchange Act de 1934 a confié à la Securities and Exchange Commission (SEC) le pouvoir de définir les normes comptables. Dès 1938, ce pouvoir a été délégué par la SEC, d’abord à la profession elle-même puis, à partir de 1973, à une entité privée indépendante, le Financial Accounting Standards Board (FASB) ${ }^{15}$.

Lorsque les missions de régulation des marchés financiers et de normalisation comptable relèvent de deux institutions non liées par une relation de contrôle, l'articulation de ces missions s'opère par le biais d'un mécanisme de représentation croisée. C’est le cas pour l'AMF et le CNC, le Président du CNC siégeant au collège de l'AMF et un représentant de l'AMF siégeant à celui du $\mathrm{CNC}^{16}$. Participant aux travaux de normalisation comptable menés par le CNC, l'AMF ${ }^{17}$ contribue ainsi à l'élaboration de la position de la place française vis-àvis des projets de normes et d'interprétations de l'IASB. Toutefois, s'agissant des entreprises cotées, les normes comptables auxquelles elles sont soumises sont les normes internationales (IAS/IFRS) et non les normes françaises. Les interventions les plus significatives de l'AMF 
dans le processus de normalisation comptable s'effectuent donc en amont de sa participation aux travaux du CNC. Au niveau européen, en tant que membre du Committee of European Regulators (CESR), elle siège comme observatrice à l'Accounting Regulatory Committee (ARC) et au niveau international, elle coopère avec ses homologues étrangers au sein de l'Organisation Internationale des Commissions de Valeurs (OICV) qui transmet à l'IASB ses commentaires sur les normes en cours d'élaboration.

C’est notamment pour permettre au normalisateur comptable français de participer plus activement à l'élaboration de ces normes comptables internationales et de donner un avis sur leur interprétation que le CNC a été une nouvelle fois réformé. L’un des enjeux majeurs de cette réforme paraît être de faire évoluer le statut du normalisateur français d'organe consultatif travaillant en amont d'une structure administrative chargée d'adopter les règlements comptables (le Comité de la Réglementation Comptable ou CRC) vers celui d'autorité administrative indépendante, statut qui est également celui de l’AMF.

\subsection{Vers une nouvelle autorité administrative indépendante ?}

Dès sa création par la loi de sécurité financière ( $1^{\mathrm{er}}$ août 2003), l’AMF a été dotée du statut d'autorité administrative indépendante (AAI) et de la personnalité morale, ce qui faisait d'elle une autorité publique indépendante $(\mathrm{APB})^{18}$. Dans ce paragraphe, nous allons d'abord nous attacher à identifier les caractéristiques qui s'attachent au statut d'AAI, forme juridique emblématique du modèle de régulateur indépendant à la française ${ }^{19}$. Nous évoquerons ensuite les vertus de l'indépendance conférée par ce statut à l'institution qui en bénéficie.

\subsubsection{De l'indépendance de la future ANC}

Le rapport Gélard (2006) consacré aux AAI situe leur origine en $1977^{20}$. Objet juridique aux contours mal définis, les AAI portent en elles «une contradiction fondamentale : la Constitution plaçant l'administration sous l'autorité du Gouvernement, comment une autorité administrative pourrait-elle en être indépendante ?». Par exception à l'article 20 de la Constitution, les AAI échappent pourtant au contrôle de l'exécutif alors même qu'elles agissent au nom de l'Etat et engagent sa responsabilité. Leur indépendance ne s’arrête d'ailleurs pas là, au moins pour celles qui sont investies d'une mission de régulation ${ }^{21}$. Dans un tel cas, elle doit également s'étendre aux entreprises du secteur régulé. Frison-Roche (rapport Gélard, 2006, tome II) souligne que «L’indépendance des Autorités tient beaucoup à 
l'indépendance de leurs membres, car de fait ce sont eux qui délibèrent et prennent les décisions imputées à l'Autorité ». Nous allons tout d'abord examiner les règles propres à garantir l'indépendance des membres des AAI. Nous envisagerons ensuite la question de l’attribution de la personnalité morale et de son impact sur l’indépendance.

Plusieurs règles se combinent pour préserver l'indépendance des membres des AAI :

- l'existence d'incompatibilités vise à prévenir les conflits d'intérêts : pour chaque AAI, certaines fonctions, de même que l'existence de liens patrimoniaux avec les entités régulées sont ainsi déclarées incompatibles avec l'appartenance à l'institution ;

- l'irrévocabilité des mandats (y compris pour faute) : elle concerne les membres des collèges des $\mathrm{AAI}$ et les protège contre d'éventuelles mesures de rétorsion de la part du Gouvernement ;

- la durée des mandats : la durée est ici comprise comme garante de compétence (et donc d'indépendance) du fait de l'apprentissage par l'expérience dont bénéficient les membres des AAI;

- l'exigence de compétence : cette règle est aujourd'hui peu fréquente sauf en ce qui concerne les exigences de recrutement de «personnalités qualifiées »; pourtant, on ne peut que se rallier à Frison-Roche lorsqu'elle soutient qu' « il serait bon que les personnes choisies pour entrer dans le collège et les personnes désignées comme Président aient avant leur désignation des compétences requises» ${ }^{22}$;

- la protection contre la capture par anticipation : deux règles se combinent ici pour protéger les membres des AAI ; le caractère non renouvelable des mandats des membres du collège organise leur protection contre les pressions de celui qui a nommé ; l'existence d’un délai de viduité interdit la reconversion des membres du collège dans une entreprise concernée par leur action passée et les soustrait ainsi à un risque de capture par les entités sur lesquelles le pouvoir est exercé ;

- le caractère collégial des décisions : en préservant l'anonymat, la collégialité empêche les tiers d’imputer la décision prise par l’Autorité à une personne précise.

Concernant l'attribution de la personnalité morale à une AAI pour la transformer en API, le rapport Gélard relève : «L'attribution de la personnalité morale à une autorité paraît illustrer, en général, la volonté du législateur d'affirmer l'indépendance de l'entité visée». Frison-Roche souligne ainsi la dimension symbolique qui s'attache à l'attribution de la personnalité morale. Dans la perspective de l'insertion de la future ANC dans le processus de 
normalisation comptable internationale, une manifestation symbolique de l'indépendance du normalisateur français contribuerait à asseoir sa légitimité sur la scène internationale.

L’indépendance des AAI possède en effet plusieurs vertus qui constituent autant de motifs de création d'un tel objet juridique. Vis-à-vis du Gouvernement, la création d'une AAI traduit souvent une défiance dans sa capacité à prendre en charge la régulation du secteur concerné, soit en raison de l'existence de conflits d'intérêts ${ }^{23}$, soit du fait de la technicité des matières traitées. Dans le cas de l'AMF, le deuxième argument joue pleinement. On peut estimer qu'il en de même vis-à-vis de la création de l’ANC. Les normes comptables ont acquis aujourd'hui une technicité telle que le modèle étatique français de normalisation n'est plus efficace ${ }^{24}$. Les compétences requises pour dialoguer avec les instances internationales de normalisation ne résident pas aujourd'hui au sein de l'Etat. Elles se trouvent dans les grands cabinets d'audit et dans les services comptables des entreprises cotées les plus importantes. L'existence d'une asymétrie d'information forte entre domaine régulé et administration joue donc en faveur de l'institution d'une AAI pour abriter l'instance française de normalisation comptable. L'adoption de cette forme institutionnelle suit donc au moins partiellement une logique d'efficacité.

Vis-à-vis des parties prenantes de l'activité régulée (dans le cas de l'ANC, les investisseurs et les autres parties prenantes de la chose comptable), l'indépendance est la condition première de la crédibilité. Cette indépendance doit «se donner à voir » (d'où les règles mentionnées plus haut) afin de susciter la confiance des parties prenantes. S’agissant d'une instance qui a vocation à participer aux négociations internationales sur les normes IFRS, sa crédibilité doit s’apprécier non seulement du point de vue des parties prenantes nationales mais également vis-à-vis des ses homologues étrangers, qu'ils agissent à l'échelon national, comme le FASB ou supranational, comme l'IASB ou l'ARC. De ce point de vue, la question de l'indépendance de l'ANC ne se résume pas aux règles garantissant celle de ses membres. Elle ne saurait être dissociée de l'examen des conditions de l’indépendance financière de l'institution.

\subsubsection{Des modalités de financement propres à renforcer l’indépendance ?}

Au stade actuel du processus de réforme du CNC, la question de son financement n’est pas à l'ordre du jour. L'article 7 du décret du 29 avril 2007 prévoit seulement que « Le Conseil National de la Comptabilité dispose de services dirigés par un directeur général». Ces services sont financés par le budget du Ministère de l'économie, des finances et de l'industrie. La question des moyens dont disposera le normalisateur comptable français se posera lors de 
la deuxième étape, celle de la création de l'ANC. A l'heure actuelle, en dehors de la prise en charge par l'Etat du coût des services administratifs, le CNC fonctionne sur le mode de « la coordination de bénévolats » (Lepetit, 2007), ce qui pose un double problème d’indépendance et de compétence.

Ainsi que le rappelle Frison-Roche (rapport Gélard, 2006, tome II), « la compétence est la garantie la plus forte de l'indépendance ». C’est la raison pour laquelle, « la question des moyens humains, comme celle des moyens budgétaires, ne doit pas être traitée séparément de la question de l'indépendance, mais conçue comme lui étant directement liée ». S'agissant d'un organisme ayant vocation à intervenir dans le processus d'élaboration des normes IFRS, l'expertise technique, au sein des services de l’ANC, des membres du pôle «normes comptables internationales" conditionnera très largement la crédibilité de l'institution sur la scène internationale. L'enjeu pour la future ANC est donc de pouvoir recruter pour sa direction générale les personnes possédant les compétences requises en leur offrant des salaires susceptibles d'entrer en compétition avec ceux du marché. Ceci suppose que soient satisfaites deux conditions: d'une part que l'ANC puisse disposer de moyens financiers suffisants et d'autre part qu'elle bénéficie d'une autonomie de gestion lui permettant notamment de recruter librement son personnel.

Dans le cas de l'AMF, ces deux conditions sont remplies. L'institution dispose tout d'abord de l'autonomie financière ${ }^{25}$ (art. L. 621-5-2 du code monétaire et financier). Ses ressources proviennent pour l'essentiel des droits versés par les personnes soumises à son contrôle $^{26}$ et accessoirement (0,2 \%) de la vente de publications ainsi que de l'organisation de journées d'étude et de formation. Pour 2007, le collège de l’AMF a approuvé un budget prévisionnel de 68 millions d’euros. L’AMF bénéfice ensuite de l'autonomie de gestion. Le Secrétaire général qui dirige les services est nommé par le collège, sur proposition du Président. C’est lui qui, dans le cadre des règles générales fixées par le collège, a qualité pour engager, gérer et licencier le personnel ainsi que pour fixer les rémunérations et les indemnités (article 30 du décret du 21 novembre 2003 relatif à l’AMF). Ainsi que le rapporte Frison-Roche, l'AMF se satisfait de sa politique salariale qui lui permet d'offrir (à quelques exceptions près) des rémunérations proches de celles du marché et ainsi de pourvoir à l'ensemble des postes vacants de l'Autorité ${ }^{27}$.

Qu'en est-il de la future ANC, s'agissant de la question de son autonomie financière ? Dans sa proposition de réforme du dispositif français de normalisation comptable, Jean-François Lepetit souligne que l'ANC «devra disposer de ressources propres et adéquates qu'il lui appartiendra de définir et dont une partie pourrait provenir du secteur 
privé ». A la manière de son homologue britannique, l'Accounting Standards Board ${ }^{28}$, l'ANC pourrait être financée à parts égales par l'Etat, les entreprises et la profession comptable. La pluralité des sources de financement combinée à l'équilibre du partage présenterait l'avantage de permettre à l'ANC de bénéficier de moyens suffisants sans pour autant dépendre d'une source de financement particulière.

Rappelons à cet égard qu'une autre AAI récente, instituée par la loi de Sécurité Financière, le Haut Conseil du Commissariat aux Comptes (H3C) souffre d'une insuffisance de moyens régulièrement dénoncée par sa Présidente ${ }^{29}$. Formellement dépendant de l'Etat pour son financement (son budget est financé par le Ministère de la Justice), le H3C est dans les faits dépendant de la profession qu'il est chargé de réguler, notamment en matière de ressources expertales dans le domaine de l'audit. Le véritable danger serait, pour l'ANC, de se trouver placée dans une situation similaire à celle du H3C faute de moyens suffisants. Dépendre techniquement et financièrement de la profession comptable libérale, et plus particulièrement des grands cabinets, et/ou de quelques grandes entreprises ferait en effet peser sinon un risque du moins un soupçon de partialité sur une institution telle que l' $\mathrm{ANC}^{30}$. C’est d'ailleurs pour un motif analogue que la loi Sarbanes-Oxley a modifié les modalités de financement du normalisateur américain ${ }^{31}$, le FASB, en transférant son financement de la profession comptable aux sociétés cotées.

La question de l'autonomie de gestion de l'ANC serait quant à elle résolue par l'attribution du statut d'API, la personnalité morale entraînant automatiquement l'autonomie de gestion. Mais, dans l'hypothèse où l'ANC serait une «simple » AAI, elle pourrait parfaitement se voir dotée d'une autonomie de gestion comme c'est aujourd'hui le cas pour la plupart des AAI.

\subsubsection{Du CNC à l'ANC : les types d'isomorphismes repérables}

Derrière les deux types d'institutions offrant une référence possible pour comprendre l'évolution du CNC, le FASB et l'AMF, se profilent en réalité deux modèles de régulateur provenant directement du monde anglo-saxon et plus précisément des Etats-Unis. Il s’agit respectivement du modèle de la Self-Regulatory Organization (SRO), ici incarné par le FASB, et de celui de l'Independent Regulatory Authority (IRA) dont la SEC constitue un exemple emblématique. Si l'on se réfère à la classification proposée plus haut (2.2.), la SRO relève plus ou moins de la régulation par la profession ${ }^{32}$, caractérisée comme on l'a vu par une forte légitimité technique et un défaut de légitimité politique. Historiquement, ce type de régulation est celui qui a prévalu aux Etats-Unis en matière de normalisation comptable ${ }^{33}$. 
C’était également le modèle dominant dans de nombreux secteurs d'activité, un modèle hérité des anciennes corporations.

Le modèle de l'IRA s'est affirmé comme alternative institutionnelle à celui de l'autorégulation, ou au moins comme complément à celle-ci lorsque l'efficacité de cette dernière s’est révélée défaillante. C’est ainsi qu'en 1934, la création de la SEC peut être comprise comme une reprise en main, à l'initiative de l'Etat fédéral, de la régulation des marchés financiers après le crack boursier de 1929. Celui-ci avait révélé des défaillances multiples des mécanismes d’autorégulation en place.

Lorsque la COB fut créée en France en 1967, c’est la SEC qui lui a servi de modèle (Frison-Roche, Rapport Gélard, 2006, tome II). Alors même que le concept d'autorité administrative indépendante n’avait pas encore été forgé, on trouvait dans cette institution les ingrédients caractéristiques des IRA américaines : collégialité, irrévocabilité des mandats des membres du collège, mandats limités dans le temps. Par la suite, la loi du 2 août 1989 a renforcé l'autonomie de la COB en ne la soumettant plus à la tutelle directe d'un ministère (Dessertine, 1997). Lorsque l’AMF a été instituée en 2003, à partir de la fusion de la COB, du Conseil des Marchés Financiers (CMF) et du Conseil de discipline de la gestion financière (CDGF), trois organismes auxquels la qualification d'AAI avait été reconnue, elle a naturellement été dotée du même statut. C’est aujourd’hui ce statut qui est envisagé pour la future ANC.

La question évidemment se pose de savoir pourquoi le législateur français, confronté à la nécessité de faire du normalisateur comptable national un interlocuteur crédible sur la scène internationale, a rejeté le modèle de la SRO associé au FASB au profit de celui du régulateur indépendant. Plusieurs éléments issus de notre cadre théorique peuvent être avancés pour tenter d'apporter une réponse.

Si l'on se réfère à la perspective du néo-institutionnalisme économique, qui met en avant le critère d'efficience comme principe de choix entre formes institutionnelles concurrentes, il est clair que l'analyse des défaillances du FASB dans la période post-Enron et les modifications apportées au financement de la FAF par la loi Sarbanes-Oxley suggèrent que ce modèle de régulation n’est pas exempt de défauts. De nombreux observateurs ont relevé la lenteur du FASB à adopter certaines normes (comptabilisation des stocks-options, entités ad hoc), alors même qu'elles visaient à améliorer la qualité de l'information financière. Dans cette perspective, le choix d'une autorité de régulation indépendante s'inscrit dans une logique d'efficience accrue en réduisant les risques de conflits d'intérêt entre le normalisateur et les parties prenantes de la comptabilité. 
A l'argument d'efficience, on peut adjoindre celui de la légitimité qui s’attache au modèle du régulateur indépendant du type IRA. Même si la SEC n’est pas exempte de critiques (Bealing et al., 1996), elle a servi de modèle à de nombreux pays lorsqu'ils ont eu à mettre en place un dispositif de régulation de leurs marchés financiers.

Enfin, on peut également lire le rejet de l'autorégulation par le législateur français comme une manifestation de dépendance de sentier, le rejet d'une normalisation comptable contrôlée par la profession s’inscrivant dans la lignée de la loi le Chapelier ${ }^{34}$ et de la suspicion d'une République restée jacobine à l'égard des organisations professionnelles à caractère corporatiste. De manière plus générale, la perte de faveur de l'endo-régulation doit être replacée dans la perspective de l'évolution à long terme des rapports entre l'Etat et les professions (Krause, 1996). S’agissant de la profession d'expert-comptable, Ramirez (2003) rapelle que la création de l’Ordre en 1942 et, surtout, son maintien en 1944 répondaient à la volonté de l'Etat d'associer la profession à un projet de rationalisation économique et sociale reposant sur la mise en œuvre d'outils macro-économiques au service de l'intervention de l’Etat. Un tel projet n'était pas compatible avec le modèle de l'endo-régulation.

Le tableau suivant fait ressortir l'ensemble de nos conclusions en les reliant à notre triple cadre théorique :

Tableau 2 : Synthèse des conclusions

\begin{tabular}{|c|c|c|}
\hline $\begin{array}{c}\text { Etapes dans l'évolution du } \\
\text { CNC }\end{array}$ & $\begin{array}{c}\text { De l'ancien CNC au nouveau } \\
\text { CNC }\end{array}$ & $\begin{array}{c}\text { Du nouveau CNC à la future } \\
\text { ANC }\end{array}$ \\
\hline $\begin{array}{l}\text { Indices d'isomorphisme } \\
\text { concurrentiel }\end{array}$ & \begin{tabular}{l}
\multicolumn{3}{l}{ Accroissement de l'efficience } \\
du CNC (taille resserrée, \\
expertise accrue des \\
commissions de travail)
\end{tabular} & $\begin{array}{l}\text { Le choix d'une AAI réduit } \\
\text { les risques de collusion avec } \\
\text { le secteur régulé ainsi que les } \\
\text { risques d'opportunisme de la } \\
\text { profession }\end{array}$ \\
\hline $\begin{array}{ll}\text { Indices } & \text { d'isomorphisme } \\
\text { mimétique } & \end{array}$ & $\begin{array}{l}\text { Abandon de la conception } \\
\text { partenariale } \\
\text { normalisation, rôle majeur } \\
\text { joué par les experts des } \\
\text { grands cabinets. }\end{array}$ & $\begin{array}{l}\text { Conformité } \text { au modèle } \\
\text { américain de l'IRA qui } \\
\text { bénéficie d'une rorte } \\
\text { légitimité sur la scène } \\
\text { internationale }\end{array}$ \\
\hline $\begin{array}{l}\text { Indices de dépendance de } \\
\text { sentier }\end{array}$ & $\begin{array}{lr}\text { Maintien } & \text { d’un } \\
\text { (consultatif) } & \text { de } \\
\text { partenariale. } & \end{array}$ & $\begin{array}{l}\text { Rejet du modèle de l'auto- } \\
\text { régulation lié à l'histoire de } \\
\text { la profession comptable } \\
\text { française }\end{array}$ \\
\hline
\end{tabular}

\section{Conclusion}

La réforme en cours du dispositif français de normalisation comptable - commencée par la réorganisation du CNC et qui, si elle est menée à son terme, devrait déboucher sur la création 
d'une Autorité des Normes Comptables (ANC) - s'inscrit en marge du sentier historique de la normalisation « à la française » et peut être considérée comme une rupture avec sa tradition partenariale. En effet, au sein du nouveau et troisième CNC, l'élaboration de la norme relève d'un organe, le collège, composé majoritairement d'experts alors que dans le premier et le deuxième CNC l'élaboration de la norme se faisait dans la cadre d'une discussion entre les représentants des diverses parties prenantes. La réforme est censée donner un surcroît de légitimité technique au normalisateur national mais sans doute au prix d’un déficit de légitimité politique.

Partant du constat de cette rupture, il nous a semblé intéressant d'en tenter une interprétation néo-institutionnelle. Les organismes de normalisation peuvent en effet être considérés comme des institutions. Ces institutions, dans leur quête de légitimité et d'efficience, sont soumises à des forces isomorphiques ${ }^{35}$ qui les dynamisent et les font évoluer de façon plus ou moins brutale. En l'occurrence, il nous a semblé que deux mimétismes pouvaient expliquer la mutation récente du CNC : le mimétisme inspiré par des organismes de normalisation anglo-saxons réputés très performants, le mimétisme inspiré par les autorités administratives indépendantes françaises incarnant le modèle français du régulateur indépendant.

Pour tester cette hypothèse, nous nous sommes livrés à une comparaison du nouveau CNC avec, d'une part, le FASB, considéré comme le normalisateur anglo-saxon de référence et, d'autre part, l'AMF considérée comme l'AAI de référence.

Il ressort de cette comparaison que le nouveau CNC est plus proche de l'AMF que du FASB. Ce qui nous conduit à penser que la future Autorité (française) des Normes Comptables (ANC) prendra la forme d'une AAI. Toutefois, le mimétisme n'est français qu'en apparence car l'AMF et avant elle la COB, en tant qu'AAI, avaient été copiées sur la SEC.

De l'ancien au nouveau CNC puis à l'ANC, l'évolution du normalisateur français fait apparaître des éléments qui relèvent de différents types d’isomorphismes. Quêtes d'efficience et de légitimité coexistent ici de même que se manifestent des formes d’héritage du passé. Mais l'histoire n'est pas finie. Les problèmes liés de l'indépendance et du financement de la nouvelle ANC ne sont pas résolus et leur résolution pourrait éventuellement s'inspirer des solutions adoptées pour l'indépendance et le financement de la SEC. Quant à la création d'un pôle « normes publiques » en son sein, elle pourrait s'inspirer de l'architecture de la FAF avec son GASB. Les ressources du mimétisme institutionnel américain ne sont donc pas épuisées.

Au plan théorique, il s'avère que les concepts de l'analyse institutionnelle se prêtent bien à l'interprétation des évolutions sur fond de mondialisation des dispositifs nationaux de 
normalisation. Ils pourraient sans doute être appliqués à l'examen de l'évolution des dispositifs d'autres pays comme par exemple la Grande-Bretagne, l’Allemagne ou le Japon.

\section{Notes}

${ }^{1}$ M. Jean-François Lepetit a fait sa carrière dans la banque (Indosuez et Paribas) avant de devenir président du Conseil des Marchés Financiers puis de la Commission des Opérations de Bourse (COB). Le 13 mars 2007, le Ministre de l'Economie, des Finances et de l'Industrie, M. Thierry Breton, le nomme à la présidence du CNC avec pour mission de "préparer une évolution de cette institution » et de «faire des propositions pour moderniser nos (celles des pouvoirs publics) méthodes de travail dans le domaine de la comptabilité » (lettre de mission du ministre en date du 21 mars 2007). Dès le 6 avril 2007, M. Jean-François Lepetit remettait au ministre son rapport avec en annexe le projet de décret réformant le CNC.

${ }^{2}$ La création de l'ANC devrait faire l'objet d'un article de la loi en préparation (avril 2008) sur la modernisation de l'économie ; les modalités de cette création feraient l'objet d'une ordonnance.

${ }^{3}$ La liste des présidents successifs des organismes français de normalisation est édifiante en ce qu'elle illustre l'évolution du dispositif français de normalisation comptable :

- Commission de normalisation des comptabilités (1946) : Robert Lacoste, député rapporteur de la Commission des fiances de l'Assemblée Nationale, et ancien ministre.

- Conseil Supérieur de la Comptabilité (1947): Francis-Louis Closon, directeur général de l’Institut National de la Statistique et des études économiques.

- Premier Conseil National de la Comptabilité (1957): Henri Montet, sous-directeur du Ministère des Finances et des Affaires Economiques ; Jean Dupont, trésorier payeur général, Yves Cotte, conseiller juridique à la Direction du Trésor.

- Deuxième Conseil National de la Comptabilité (1996) : Georges Barthès de Ruyter, ancien associé d'Arthur Andersen et ancien président de l'IASC ; Antoine Bracchi, ancien associé et président d'Ernst \&Young France ; Jean-François Lepetit, ancien banquier (voir note $n^{\circ} 1$ ).

${ }^{4}$ Cette obligation faite à son Président de se consacrer exclusivement au CNC a été supprimée par un décret du 13 mars 2007 (n²007-336).

${ }^{5}$ Le ministre chargé de l'Economie ou son représentant, président ; le garde des sceaux, ministre de la Justice, ou son représentant, vice-président ; le ministre chargé du Budget ou son représentant ; un membre du Conseil d’Etat, désigné par le vice-président de celui-ci, un membre de la Cour des Comptes, nommé par le premier président de celle-ci, et un membre de la Cour de Cassation, désigné par le premier président de celle-ci et le procureur général.

${ }^{6} \mathrm{La}$ «professionnalisation » du deuxième CNC sera symboliquement marquée par la nomination à sa tête de Georges Barthès de Ruyter, ancien associé d’Arthur Andersen et ancien président de l’IASC.

${ }^{7}$ Le règlement du Parlement européen et du Conseil du 19 juillet 2002 (CE n 1606/2002) a confirmé ce choix en exigeant que les sociétés européennes faisant appel public à l'épargne préparent à partir de 2005 leurs comptes consolidés en conformité avec les normes de l’IASB.

${ }^{8}$ Un arrêté du 19 novembre 2007 a nommé les neuf premiers membres du Collège ainsi que les membres du comité concultatif.

${ }^{9}$ La nouvelle économie institutionnelle n'a guère manifesté d’intérêt, elle non plus, pour cette question à la notable exception de North dont l'un des apports majeurs réside précisément dans l'élaboration d'une théorie du changement institutionnel.

10 «Une institution est une idée d'œuvre ou d'entreprise qui se réalise et dure juridiquement dans un milieu social ; pour la réalisation de cette idée, un pouvoir s’organise qui lui procure des organes ; d'autre part, entre les membres du groupe social intéressé à la réalisation de l’idée, il se produit des manifestations de communion dirigées par les organes du pouvoir et réglées par des procédures » (Hauriou, 1925).

${ }^{11}$ Le FASB peut d'ailleurs être considéré, dans une perspective dynamique, comme le produit de l'évolution d'une institution qui relevait plutôt du type de l'endo-régulation. Ce sont les réformes successives de l'institution américaine de normalisateur comptable qui ont conduit à la structure actuelle du FASB, conforme au type technocratique du régulateur indépendant (Zeff, 2003). On pourrait faire une observation analogue au sujet de l'évolution du normalisateur comptable britannique.

12 « Tout membre du collège doit informer le Président :

- des fonctions économiques ou financières qu’il a exercées au cours des deux années précédant sa nomination, qu'il exerce ou qu'il s’apprête à exercer ; 
- de tout mandat de direction, d'administration, de surveillance ou de contrôle qu'il a détenu au sein d'une personne morale au ours des deux années précédent sa nomination, qu'il détient ou s'apprête à détenir.

Ces informations, ainsi que celles concernant le Président, sont tenues à la disposition des membres du collège ».

${ }^{13}$ L'arrêté du 19 novembre 2007 (JO n 277 du 29 novembre 2007, p. 19406) du Ministre de l'économie, des finances et de l'emploi portant nomination au collège et au comité consultatif du nouveau CNC révèle l'interprétation qui a été faite de cet article et, en même temps, ses limites (voir note 27).

${ }^{14}$ Afin de prévenir tout risque de conflit d'intérêt lié à l'obligation pour les entreprises d'acquérir le texte des normes comptables, la loi Sarbanes-Oxley a cependant exigé du FASB qu'il mette en ligne gratuitement sur son site (www.fasb.org) ses Statements of Financial Accounting Standards ainsi que les Concepts Statements.

${ }^{15}$ Il n'est pas question de passer ici en revue l'histoire mouvementée des relations entre la SEC et le FASB. Il est intéressant de noter toutefois que la SEC a conservé un pouvoir d'influence significatif sur les normes comptables américaines. Ainsi que le rappelle Cormier (2002), le régulateur boursier enrichit souvent les travaux du FASB. Souvent, ses exigences précèdent les normes du FASB et dans quelques cas, il est arrivé qu'il contredise carrément une norme du FASB, obligeant ce dernier à revoir sa copie.

${ }^{16}$ De la même manière, au Royaume-Uni, une représentation croisée a été mise en place entre la Financial Services Authority (FSA) et le Financial Reporting Council (FRC), institutions respectivement chargées de la régulation des marchés financiers et de la gouvernance d'entreprise (y compris les règles relatives à l'information financière publiée par les entreprises).

${ }^{17}$ Plus précisément, au sein de l'AMF, c'est la direction des affaires comptables qui a vocation à interagir avec le CNC et notamment son département doctrine comptable, chargé de définir les positions doctrinales de l'AMF pour la bonne application des normes comptables.

${ }^{18}$ En droit français, les établissements publics sont des organismes dotés de la personnalité morale mais soumis à la tutelle d'un ministère. A partir de 2003, le législateur a choisi de doter de la personnalité morale des autorités indépendantes, c'est-à-dire libres de toute tutelle administrative. Il a recouru pour ce faire à l'appellation nouvelle d'autorité publique indépendante (Rapport Gélard, 2006).

${ }^{19}$ La question de l'attribution de la personnalité morale au régulateur, un choix qui ne concerne aujourd'hui que quatre instances sur un total de 39 AAI recensées en 2006, sera également évoquée.

${ }^{20}$ Celle-ci se confond avec la création de la Commission Nationale de l'Informatique et des libertés (CNIL).

${ }^{21} \mathrm{Au}$ sein des AAI, on distingue classiquement deux catégories: celle des régulateurs économiques (Commission de régulation de l'Energie, Commission bancaire,...) et celle des Autorités protectrices des libertés publiques et des personnes (Conseil Supérieur de l'Audiovisuel, Haute Autorité de Lutte contre les Discriminations et pour l'Egalité).

${ }^{22}$ De ce point de vue, la composition du collège du CNC rénové dont on peut penser qu'elle préfigure celle de l'ANC, fait une large place (9 sur les 16 membres) à des «personnes désignées, à raison de leur compétence économique et comptable, (...), parmi lesquelles le ministre chargé de l'économie nomme le président ».

${ }^{23}$ C'est le cas chaque fois qu'il existe une entreprise publique dans un secteur ouvert à la concurrence ou plus généralement soumis à régulation.

${ }^{24}$ A cet égard, voir l'appel à réformer le CNC lancé dans Les Echos (16 mars 2007) par E. Boris et C. Lopater (membre du CNC), dressant un constat sévère: "La France n’a pas tiré les conséquences de la nouvelle compétition intellectuelle mondiale, déterminante sur les choix de réglementation comptable " et "Seule la création d'un comité permanent IFRS dédié à cela, composé de membres actifs, compétents, spécialisés en IFRS,(...) dans un cadre juridique bien défini et une gouvernance adaptée, répondra à ces insuffisances ».

${ }^{25}$ L'autonomie financière est une conséquence directe de la personnalité morale. Toutefois, il est possible de doter une AAI de l'autonomie financière sans pour autant lui attribuer la personnalité morale. C'était le cas de la COB (rapport Gélard, 2006, p. 68).

${ }^{26}$ Le budget de l'AMF est arrêté par le collège sur proposition du secrétaire général. Les droits versés par les personnes soumises à son contrôle concernent par exemple l'examen de l'obligation de dépôt d'une offre publique ou le contrôle d'un document de référence annuel. Le montant des droits et contributions perçus par l'AMF est fixé par décret, pris après avis du collège, dans les limites fixées par la loi (art. L. 621-5-2 et L. 621-53 du code monétaire et financier).

${ }^{27}$ Le nombre des collaborateurs de l’AMF rattachés au secrétariat général est de 360 en 2007.

${ }^{28}$ L'ASB est financé par le Financial Reporting Council (FRC) auquel il est rattaché. C'est en réalité le FRC qui bénéficie d'un financement partagé par l'Etat, les entreprises et la profession comptable.

${ }^{29}$ Dans le dernier rapport annuel publié (rapport 2006 paru le $1^{\mathrm{er}}$ août 2007), la Présidente qualifie de « critique » la situation dans laquelle se trouve le $\mathrm{H} 3 \mathrm{C}$ et indique que le manque de moyens a contraint le Haut Conseil à privilégier sa mission de normalisation au détriment des autres missions (contrôle de qualité, discipline notamment). 


\begin{abstract}
${ }^{30}$ La nomination (arrêté du 19 novembre 2007), parmi les huit membres « opérationnels »du Collège, de trois grands professionnels en activité dans les grands cabinets est évidemment de nature à nourrir ce soupçon de dépendance sinon financière du moins doctrinale.

${ }^{31}$ Les auditions réalisées par le Congrès après l’affaire Enron fournissent à cet égard des éléments édifiants. Par exemple, le témoignage d'Arthur Levitt, ancien Président de la SEC, évoquant (notre traduction) « le fait que le Board est financé par les entreprises mêmes auxquelles sont destinées les normes qu'il édicte, qui reviennent souvent le voir en disant : 'Si vous adoptez cette norme, nous vous couperons les vivres'. Ceci est mal. Nous devons modifier leur mode de financement " ( the fact that the board is funded by the very firms for whom they set standards, who often come back to them and say, 'If you're going to set this standard, we're going to cut off your funding.' That's wrong. We've got to change their funding”). Ce témoignage est accessible à l'adresse suivante : http://www.senate.gov/ gov_affairs/012401witness.htm

${ }^{32}$ Si l'on y regarde de plus près, la régulation comptable est en réalité partagée puisque la SEC exerce un contrôle ultime sur les normes produites par le FASB. Cependant, ce contrôle n’a pas toujours été d'égale intensité au fil du temps. Par ailleurs, le FASB est parfois l'objet de pressions politiques intenses de la part du Congrès, tendant à le dissuader d'adopter des normes contraires à l'intérêt de certains lobbies (par exemple, en 2004, lorsque le FASB a proposé de comptabiliser les stock-options comme une charge).

${ }^{33}$ De 1938 à 1972, la SEC a délégué son pouvoir de normalisation à la profession (AICPA). En 1972, sur la recommandation d'un ancien membre du collège de la SEC, Francis Wheat, qui présidait le comité de l'AICPA, le FASB fut institué au sein de la FAF en tant que normalisateur indépendant (Block, 2003).

${ }^{34}$ Cette loi, votée le 14 juin 1791 et qui porte le nom du constituant qui en fit la proposition, limitait les droits d'association et mettait fin au système des corporations.

${ }^{35}$ Notons que l'isomorphisme mimétique évoqué ici n’est pas le seul processus impliqué dans la tendance à l'homogénéisation des politiques publiques : les phénomènes d'isomorphismes coercitif (par exemple liés à l'obligation de transposer une directive européenne dans les droits nationaux des pays membres) et normatif (suscité par la diffusion des « bonnes pratiques » via certaines professions) interviennent également dans cette évolution
\end{abstract}

\title{
Bibliographie
}

BEALING, W.E., DIRSMITH, M.W., FOGARTY, T.J. (1996), « Early regulatory actions by the SEC: an institutional theory perspective on the dramaturgy of political exchanges", Accounting, Organizations, and Society, Vol. 21 No.4, pp.317-38.

BENSEDRINE J. et DEMIL B. (1998), « L'approche néo-institutionnelle des organisations » in LAROCHE H. et NIOCHE J-P., Repenser la stratégie, Vuibert, pp. 85-110.

BLOCK C.D. (2003), « Congress and Accounting Scandals: Is the Pot Calling the Kettle back? », Nebraska Law Review, vol. 82, pp. 365-459.

BOTZEM S. et QUACK S. (2006), "Contested rules and shifting boundaries: International standard setting in accounting ", in M-L. DJELIC et K. SAHLIN-ANDERSSON (eds.), Transnational governance: Institutional dynamics of regulation, Cambridge University Press.

BRILOFF A.J. (1982), La 3ème colonne : la face cachée de la comptabilité des entreprises, Londreys ; trad.de l'américain : (1981), The truth about corporate accounting, Harper and Row.

CHANTIRI-CHAUDEMANCHE R. (2004), " La normalisation comptable et ses acteurs », Revue Sciences de Gestion, $n^{\circ}$ 43, p. 51-74.

CHIAPELLO E. (2005), Les normes comptables comme institutions du capitalisme. Une analyse du passage aux normes IFRS à partir de 2005, Sociologie du travail, $n^{\circ} 3$, pp. 362382.

CHIAPELLO E. et MEDJAD K. (2007), « Une privatisation inédite de la norme : le cas de la politique comptable européenne, Sociologie du travail, n 49, pp. 46-64.

COASE R. (1937), The Nature of the Firm, Economica, Vol. 4, n 16, pp. 386-405.

COLASSE B. et STANDISH P. (1998), « De la réforme 1996-1998 du dispositif français de normalisation comptable », Comptabilité-Contrôle-Audit, tome 4, vol. 2, pp. 5-27.

COLASSE B. (2004), Harmonisation comptable internationale : la résistible ascension de l’IASC/IASB, Gérer et Comprendre, nº 75, pp. 30-40. 
COLASSE B. (2005), " La régulation comptable entre privé et public », in Capron M., Les normes comptables internationales, instrument du capitalisme financier, La découverte, pp. 27-48.

CORMIER D. (2002), Comptabilité anglo-saxonne et internationale, Economica.

DELMAS-MARTY M. (2004), Le relatif et l'universel, Seuil.

DESSERTINE P. (1997), «La commission de contrôle des marchés dans la normalisation comptable », Comptabilité-Contrôle-Audit, tome 3, vol. 1, pp. 69-87.

DURKHEIM E. (1993), Les règles de la méthode sociologique, Presses Universitaires de France.

DIMAGGIO P. et POWELL W. (1983), « The Iron Cage Revisited: Institutional Isomorphism and Collective Rationality in Organizational Field », American Sociological Review, 48, pp. 147-160.

DIMAGGIO P. and POWELL W. (1991), "Introduction." in Powell W. and DiMaggio P., The New Institutionalism in Organizational Analysis, University of Chicago Press, pp. 1-38.

DJELIC M-L. et QUACK S. (2007), « Overcoming path dependency - Path generation in open systems », Theory and Society, vol. 36, n 2, pp. 161-186.

GELARD P. (2006), Rapport sur les autorités administratives indépendantes, Office parlementaire d'évaluation de la législation, 2 tomes.

HALL P.A. et SOSKICE D. (2001), Varieties of capitalism: The institutional foundations of comparative advantage, Oxford, Oxford University Press.

HAURIOU M. (1925), « La théorie de l'institution et de la fondation. Essai de vitalisme social » in Aux sources du droit: le pouvoir, l'ordre et la liberté, Cahiers de la Nouvelle Journée, $n^{\circ} 23$ (réimprimé Université de Caen, 1990).

KRAUSE E.A. (1996), Death of the guilds: professions, states, and the advance of capitalism, 1930 to the present, New Haven, Conn: Yale University Press.

LA PORTA R., LOPEZ-DE-SILANES F., SHLEIFER A. et R. VISHNY (1998), « Law and Finance », Journal of Political Economy, 106, pp. 1113-55.

MEYER J. et ROWAN B. (1977), « Institutionalized Organizations: Formal Structure as Myth and Ceremony », American Journal of Sociology, 83, pp. 333-63.

MILLARD E. (1995), “ Hauriou et la théorie de l’institution”, Droit et Société, n 30/31, pp. 381-412.

NORTH D. (1990), Institutions, Institutional Change and Economic Performance, Cambridge University Press.

OFFE C. (1996), « Designing institutions in East European transitions », in R. E. Goodin, ed, The Theory of Institutional Design, Cambridge University Press.

POCHET C. (2007), « La régulation de la profession d'auditeur en France et aux Etats-Unis : une étude comparée du H3C et du PCAOB », Revue Française de Gouvernance d'Entreprise, $\mathrm{n}^{\circ}$ 1, pp. 93-111.

RADAELLI C.M. (2000), «Policy Transfer in the European Union: Institutional Isomorphism as a Source of Legitimacy », Governance, vol. 13, n 1, pp. 25-43, 2000.

RAMIREZ C. (2003), " Du commissariat aux comptes à l'audit : les Big 4 et la profession comptable depuis 1970 », Actes de la Recherche en Sciences Sociales, n 146-147, pp.62-79.

SCHNEIDER V. (1998), " Europeanization and the re-dimensionalization of the public sector: Telecommunications in Germany, France and Italy ", Paper presented at the Conference on Europeanization and Domestic Political Change, European University Institute, Florence, 19-20 June.

WEIR M. and SKOCPOL T. (1985), « State Structures and the Possibilities for "Keynesian" Responses to the Great Depression in Sweden, Britain, and the United States ». in Evans P.B., 
RUESCHEMEYER D. and SKOCPOL T. (eds.), Bringing the State Back In, Cambridge University Press, pp. 107-168

WILLIAMSON O. (1985), The Economic Institutions of Capitalism, The Free Press, New York.

YOUNG J. (1994), " Outlining space: Agenda issues and the FASB », Accounting, Organization and Society, vol. 19, $\mathrm{n}^{\circ} 1$, pp. 83-109.

ZEFF S.A. (2003), " How the US accounting profession got where it is today: Part I », Accounting Horizons, vol. 17, $\mathrm{n}^{\circ}$ 3, pp.189-205.

\section{Webographie}

CNC : www.minefi.gouv.fr/directions_services/CNCompta/

FASB : www.fasb.com

\section{Documentation}

Proposition de réforme du dispositif français de normalisation comptable, Jean-François Lepetit, 6 avril 2007, 9 p.

Décret n 2007-336 du 13 mars 2007 modifiant le décret n 96-749 du 26 août 1996 relatif au Conseil National de la Comptabilité.

Arrêté du 13 mars 2007 portant nomination du président du Conseil National de la Comptabilité.

Décret n 2007-629 du 27 avril 2007 relatif au Conseil National de la Comptabilité.

Arrêté du 19 novembre 2007 portant nomination au Conseil National de la Comptabilité.

CNC Collège, procès-verbal de la séance du mercredi 19 décembre 2007 (composition des commissions, nomination du directeur général,...), document nº1_07_02. 